\title{
水解制氢材料研究进展
}

\author{
双雯峰 ${ }^{1,2}$, 陈顺鹏 ${ }^{1}$, 武晓娟 ${ }^{1}$, 郑 捷 ${ }^{1}$, 李星国 ${ }^{1}$ \\ (北京大学 1. 化学与分子工程学院; 2. 前沿交叉学科研究院, 北京 100871)
}

摘 要: 水解制氢是一种常温常压下的现场制氢方式。由于水解制氢材料氢含量高, 储存容易, 运输方便, 安全可靠, 一直受到研究者们的关注。本文综述了近年来水解制氢材料的总体发展情况, 介绍了三类主要的水解制氢材料, 包 括嗍氢化物 $\left(\mathrm{NaBH}_{4}, \mathrm{NH}_{3} \cdot \mathrm{BH}_{3}\right)$ 、金属 $(\mathrm{Mg}, \mathrm{Al})$ 以及金属氢化物 $\left(\mathrm{MgH}_{2}\right)$, 对不同材料的制氢原理、主要问题、催化剂 与材料设计进行了详细介绍, 比较了不同体系的特点与制氢成本, 并对水解制氢及水解制氢材料的现状和商业化 面临的困难做了评价, 最后对未来的发展方向进行了展望。

关 键 词: 制氢; 水解; 金属; 金属氢化物; 嗍氢化物; 制氢成本

中图分类号: TQ174 文献标识码: A

\section{Recent Progress on Materials for Hydrogen Generation via Hydrolysis}

\author{
DENG Jifeng ${ }^{1,2}$, CHEN Shunpeng ${ }^{1}$, WU Xiaojuan ${ }^{1}$, ZHENG Jie ${ }^{1}$, LI Xingguo ${ }^{1}$
}

(1. College of Chemistry and Molecular Engineering, Peking University, Beijing 100871, China; 2. Academy of Advanced Interdisciplinary Studies, Peking University, Beijing 100871, China)

\begin{abstract}
Hydrolysis is a unique method for hydrogen generation at ambient condition. Widespread attentions have been paid to materials for hydrogen generation via hydrolysis due to several advantages: high theoretical hydrogen capacity, moderate storage and operation condition, safety, etc. In this paper, recent progress and development in this area were reviewed. Three types of materials including borohydride $\left(\mathrm{NaBH}_{4}, \mathrm{NH}_{3} \cdot \mathrm{BH}_{3}\right)$, metal $(\mathrm{Mg}, \mathrm{Al})$, and metal hydride $\left(\mathrm{MgH}_{2}\right)$ were introduced. Several issues about them were discussed specifically: mechanism, main problems, designments of catalysts and materials, etc. Based on these discussions, we compared the different materials mentioned above, commented their current performances and practical difficulties. At last, prospects in this field were presented.
\end{abstract}

Key words: hydrogen generation; hydrolysis; metal; metal hydride; borohydride; cost of hydrogen

伴随着经济发展和极端天气增多, 全球能源的 消耗量也在逐年增长, 随之带来的气候变化又进一 步加剧了能源的消耗。发展新型可再生的清洁能源 是解决环境和能源危机的必然选择。作为清洁、高 效的可再生能源, 氢在新能源领域的发展和应用中
具有重要地位，是一种理想的能量载体，能够整合 利用全球丰富的能源资源 ${ }^{[1-5]}$ 。基于此的氢能源和 “氢经济”是极具潜力的未来能源运行模式。

在众多储氢材料中, 水解制氢材料属于不可逆 储氢材料。它通过水解反应, 释放氢气, 适合在用氢

收稿日期：2020-04-22；收到修改稿日期：2020-07-10；网络出版日期：2020-08-01

基金项目: 科技部十三五专项(2018YFB1502102); 国家自然科学基金面上项目(51971004, 51771002)

Ministry of Science and Technology (2018YFB1502102); National Natural Science Foundation of China (51971004, 51771002)

作者简介: 邓雯峰(1995-), 男, 博士研究生. E-mail: jfdeng@pku.edu.cn DENG Jifeng(1995-), male, PhD candidate. E-mail: jfdeng@pku.edu.cn

通信作者: 李星国，教授.E-mail: xgli@pku.edu.cn LI Xingguo, professor. E-mail: xgli@pku.edu.cn 
现场制氢。水解制氢具有如下优势: 1)质量储氢密度 和体积储氢密度均较高, 如表 1 所示, $\mathrm{NaBH}_{4} 、 \mathrm{MgH}_{2}$ 等体系理论质量储氢密度超过 $6 \%$; 同时这类材料 的体积储氢密度也高于商用的高压储氢；2)水解制 氢是自发的放热反应, 在常温常压下进行, 制氢装 置相对简单; 3)水解制氢材料容易保存, 相对安全, 便于存储和运输；4)反应副产物基本无毒无害, 符 合绿色化学的要求。

基于以上特点, 水解制氢极具应用潜力。但在 实用化过程中, 仍然存在许多问题, 一定程度上限 制了其发展, 具体如下: 1)制氢成本较高, 高含氢量 水解制氢材料制备过程复杂且能耗较高, 总体能效 低; 2)材料体系面临表面钝化、催化剂失活等问题; 3)水解实际耗水量远超理论耗水量, 限制了总体储 氢密度。按照理论耗水量进行反应的研究鲜有报道; 4)反应控制需要配套装置 ${ }^{[7-10]}$, 涉及固相传质、三相 分离等工程问题; 5) 水的液相温度区间限制了水解 所适用的温度范围。

近年来国内外关于水解制氢材料的相关研究主 要围绕水解反应过程、水解材料及催化剂设计、材 料制备方法以及制氢装置开发等方面展开。本文将 常见的水解制氢材料, 按照不同的化学性质划分为 金属、金属氢化物和嗍氢化物三大类, 分别进行介 绍。不同类型材料面临的关键性问题不同, 本文按 照其特点综述了近期的研究进展。

\section{1 嗍氢化物水解制氢材料}

研究最广也最具吸引力的嗍氢化物水解制氢
材料是 $\mathrm{NaBH}_{4}$ 和 $\mathrm{NH}_{3} \cdot \mathrm{BH}_{3}$ ，此外还包括 $\mathrm{LiBH}_{4}{ }^{[11]}$ 、 $\mathrm{Mg}\left(\mathrm{BH}_{4}\right)_{2}$ 和 $\mathrm{N}_{2} \mathrm{H}_{4} \cdot \mathrm{BH}_{3}{ }^{[12]}$ 等。 $\mathrm{B}$ 与 $\mathrm{H}$ 的电负性相差 不足 0.2 , 因此硼氢化物中 $\mathrm{B}$ 与 $\mathrm{H}$ 以共价键结合, 再 与阳离子配位形成离子键 $\left(\right.$ 如 $\mathrm{NaBH}_{4}, \mathrm{LiBH}_{4}$ )或者形 成共价键(如 $\mathrm{NH}_{3} \cdot \mathrm{BH}_{3}, \mathrm{~N}_{2} \mathrm{H}_{4} \cdot \mathrm{BH}_{3}$ ) 构成嗍氢化物。 嗍氢化物化学稳定性和热稳定性良好, 能够在室温 环境下长期保存, 在水和部分极性溶剂中具有较高 的溶解度, 在碱性溶液中能够稳定存在。下面介绍 最典型的配位氢化物 $\mathrm{NaBH}_{4}$ 和分子型氢化物 $\mathrm{NH}_{3} \cdot \mathrm{BH}_{3}$ 。

\section{$1.1 \mathrm{NaBH}_{4}$ 水解制氢}

$\mathrm{NaBH}_{4}$ 是研究最广泛的水解制氢材料, 其水解 制氢过程需要进行催化。总反应如式(1)所示。

$\mathrm{NaBH}_{4}+(2+x) \mathrm{H}_{2} \mathrm{O}=\mathrm{NaBO}_{2} \cdot x \mathrm{H}_{2} \mathrm{O}+4 \mathrm{H}_{2}(x=2$ or 4$)$

所涉及催化剂包括两类一均相催化剂和异相 催化剂, 催化机理如图 1 所示 ${ }^{[13]}$, 已通过同位素示 踪验证。对于均相催化, 催化剂酸和 $\mathrm{NaBH}_{4}$ 均溶于 水中, $\mathrm{BH}_{4}{ }^{-}$与 $\mathrm{H}_{3} \mathrm{O}^{+}$结合放出 $\mathrm{H}_{2}$, 并与水结合形成水 合嗍烷, 重复上述过程, 最终生成水合偏硼酸和 $\mathrm{H}_{2}$ 。异相催化则将固态的催化剂浸泡在 $\mathrm{NaBH}_{4}$ 水溶 液中, $\mathrm{BH}_{4}{ }^{-}$在催化剂表面解离生成原子态的吸附 $\mathrm{H}$ 。催化剂同时获取电子, 用于解离水, 并生成 $\mathrm{BH}_{3}(\mathrm{OH})^{-}$, 重复上述步骤最终生成 $\mathrm{H}_{2}$ 和 $\mathrm{B}(\mathrm{OH})_{4}{ }^{-}$。 由于均相催化控制困难, 实际体系一般采用异相催 化 $^{[14]}$ 。

异相催化剂包括 Pt、Ru 等贵金属催化剂 ${ }^{[15-19]}$, 以及 $\mathrm{Fe} 、 \mathrm{Co} 、 \mathrm{Ni}$ 等非贵金属催化剂 ${ }^{[20-26]}$ 。在所有 催化剂中, Co 基催化剂综合性能优异且成本相对较 低, 是 $\mathrm{NaBH}_{4}$ 水解研究中最常用的催化剂之一。

表 1 常见水解制氢材料的基本性能参数

Table 1 Fundamental performances of materials for hydrogen generation via hydrolysis

\begin{tabular}{|c|c|c|c|c|c|c|}
\hline Material & Molar mass $/\left(\mathrm{g} \cdot \mathrm{mol}^{-1}\right)$ & Density $/\left(\mathrm{g} \cdot \mathrm{cm}^{-3}\right)$ & $\Delta H^{[6]} /\left(\mathrm{kJ} \cdot \mathrm{mol}^{-1}{ }_{\mathrm{H}_{2}}\right)$ & $C_{\mathrm{M}\left(\mathrm{H}_{2}\right)} * / \mathrm{wt} \%$ & $C_{\mathrm{M}\left(\mathrm{H}_{2}\right)}\left(\mathrm{H}_{2} \mathrm{O}\right)^{\#} / \mathrm{wt} \%$ & $\mathrm{C}_{\mathrm{V}\left(\mathrm{H}_{2}\right)}{ }^{\dagger} /\left(\mathrm{g}_{\mathrm{H}_{2}} \cdot \mathrm{L}^{-1}\right)$ \\
\hline $\mathrm{NaBH}_{4}$ & 37.83 & 1.07 & -47.15 & 21.32 & 7.34 & 228.09 \\
\hline $\mathrm{NH}_{3} \cdot \mathrm{BH}_{3}$ & 30.86 & 0.78 & -75.67 & 19.50 & 7.05 & 151.60 \\
\hline $\mathrm{Mg}$ & 24.31 & 1.74 & -352.90 & 8.29 & 3.34 & 144.16 \\
\hline $\mathrm{Ca}$ & 40.08 & 1.55 & -413.60 & 5.03 & 2.65 & 77.97 \\
\hline $\mathrm{Al}$ & 26.98 & 2.70 & -284.40 & 11.21 & 3.73 & 302.62 \\
\hline $\mathrm{LiH}$ & 7.95 & 0.82 & -111.20 & 25.36 & 7.06 & 207.97 \\
\hline $\mathrm{NaH}$ & 24.00 & 1.40 & -83.70 & 8.40 & 4.80 & 117.60 \\
\hline $\mathrm{KH}$ & 40.02 & 1.43 & -81.10 & 5.04 & 3.47 & 72.04 \\
\hline $\mathrm{MgH}_{2}$ & 26.32 & 1.45 & -138.80 & 15.32 & 6.47 & 222.12 \\
\hline $\mathrm{CaH}_{2}$ & 42.09 & 1.70 & -116.05 & 9.58 & 5.16 & 162.84 \\
\hline $\mathrm{AlH}_{3}$ & 30.00 & 1.49 & -126.87 & 20.16 & 7.20 & 300.34 \\
\hline
\end{tabular}

\footnotetext{
* $C_{\mathrm{M}\left(\mathrm{H}_{2}\right)}:$ Mass of $\mathrm{H}_{2}$ released/mass of material

${ }^{*} C_{\mathrm{M}\left(\mathrm{H}_{2}\right)}\left(\mathrm{H}_{2} \mathrm{O}\right)$ : Mass of $\mathrm{H}_{2}$ released/(mass of material + mass of water)

${ }^{\dagger} C_{\mathrm{V}_{\left(\mathrm{H}_{2}\right)}}$ : Mass of $\mathrm{H}_{2}$ released/(mass of material/density)
} 
针对 Co-B 催化剂的研究, Patel 等 ${ }^{[27]}$ 的综述已经有 了较好的总结, 包括前驱体、制备条件、其他元素 掺杂对催化剂结构和性能的影响。

目前，催化剂的性能已经基本满足制氢速率的 要求。在实际体系中, 需要反复使用催化剂, 必须进 行有效负载。图 2 展示了常用的载体类型, 主要包 括泡沫镍 ${ }^{[25,28]} 、 \mathrm{Ti}$ 网 ${ }^{[26]}$ 等金属基载体, 在气体净化中 经常使用的硅酸盐、铝酸盐类蜂窝陶瓷载体 ${ }^{[29-30]}$, 以 及包括水凝胶在内的聚合物载体 ${ }^{[31-35]}$ 。载体除了满 足固定、分散催化剂等要求外, 在体系中还要求避 免偏硼酸盐的堆积以防止催化剂钝化 ${ }^{[30]}$ 。上述载体 均拥有宏观多孔的结构, 保证了反应的有效传质。 为保证体系的质量储氢量, 载体应当尽量轻质, 这 一方面聚合物相比金属和陶瓷载体更具优势。

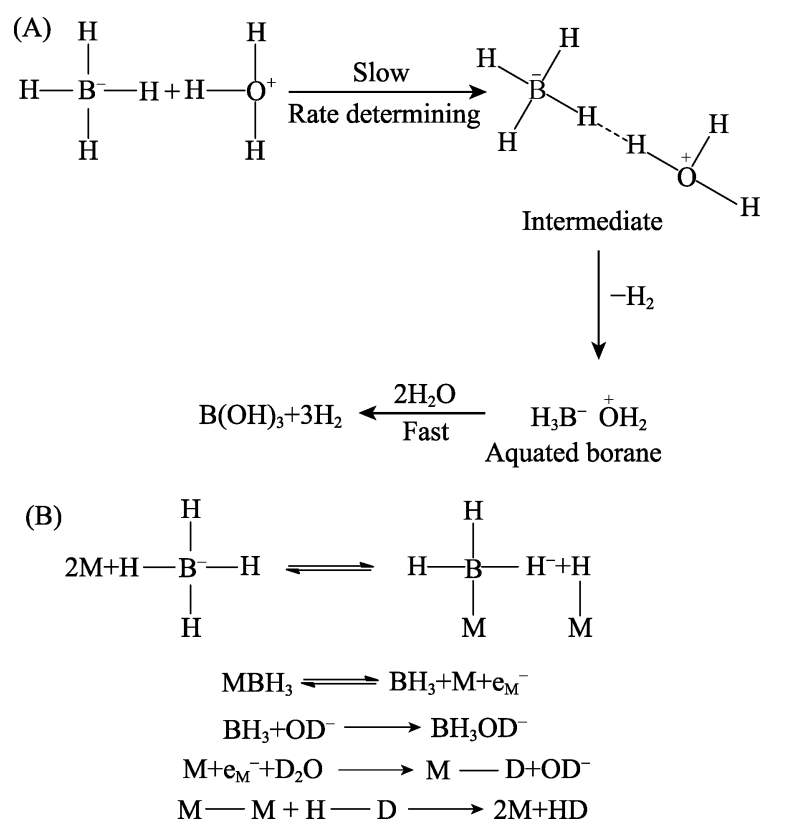

图 $1 \mathrm{NaBH}_{4}$ 的均相催化(A)和异相催化(B)水解反应机理 ${ }^{[13]}$ Fig. 1 Schemes for the mechanisms of homogeneous (A) and heterogeneous (B) hydrolysis of $\mathrm{NaBH}_{4}{ }^{[13]}$

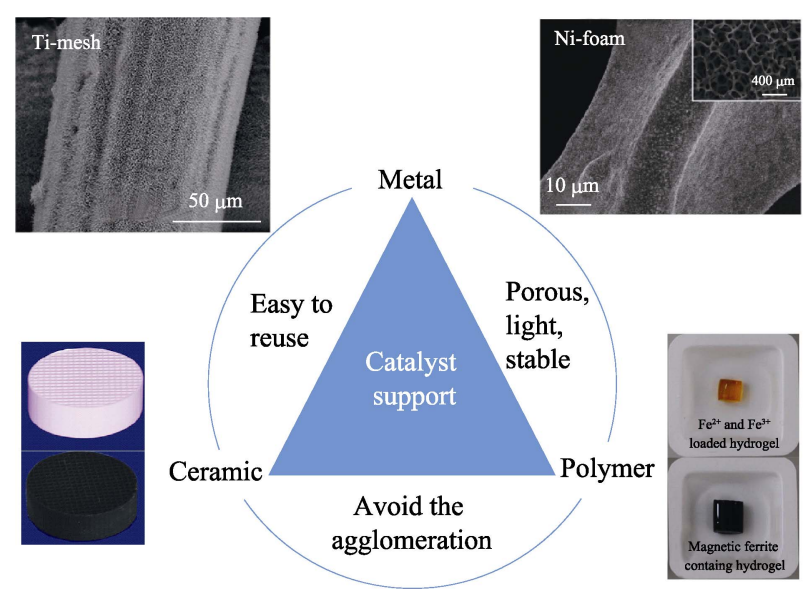

图 $2 \mathrm{NaBH}_{4}$ 水解催化剂常用载体 ${ }^{[25-26,28-35]}$

Fig. 2 Supports for catalysts of $\mathrm{NaBH}_{4}$ hydrolysis ${ }^{[25-26,28-35]}$
工业上采用 Bayer 法或 Brown-Schlesinger 法 ${ }^{[36]}$ 制备 $\mathrm{NaBH}_{4}$ ，总反应如式(2 3)所示。

Brown-Schlesinger 法:

$$
4 \mathrm{NaH}+\mathrm{B}\left(\mathrm{OCH}_{3}\right)_{3}=\mathrm{NaBH}_{4}+3 \mathrm{NaOCH}_{3}
$$

Bayer 法:

$\mathrm{Na}_{2} \mathrm{~B}_{4} \mathrm{O}_{7}+16 \mathrm{Na}+8 \mathrm{H}_{2}+7 \mathrm{SiO}_{2}=4 \mathrm{NaBH}_{4}+7 \mathrm{Na}_{2} \mathrm{SiO}_{3}$

反应以 $\mathrm{B}\left(\mathrm{OCH}_{3}\right)_{3}$ 或 $\mathrm{Na}_{2} \mathrm{~B}_{4} \mathrm{O}_{7}$ 为原料, 采用 $\mathrm{NaH}$ 或 $\mathrm{Na} 、 \mathrm{H}_{2}$ 等还原剂, 在高温(Bayer 法需要高压)下 进行。反应过程控制复杂，耗能巨大，导致 $\mathrm{NaBH}_{4}$ 制备成本较高。Kojima 等 ${ }^{[37]}$ 发展了以 $\mathrm{NaBO}_{2}$ 和 $\mathrm{MgH}_{2}$ 为原料的高温热合成法, 能将水解副产物 $\mathrm{NaBO}_{2}$ 直接用于合成 $\mathrm{NaBH}_{4}$ 。朱敏和欧阳柳章课题 组 ${ }^{[38]}$ 在此基础上, 以 $\mathrm{NaBO}_{2}$ 和 $\mathrm{MgH}_{2}$ 为原料, 通过 氢压反应球磨，实现了 $89 \%$ 的产率。随后，Ouyang 等 ${ }^{[39]}$ 直接以 $\mathrm{NaBO}_{2} \cdot x \mathrm{H}_{2} \mathrm{O}$ 和 $\mathrm{Mg}$ (过量)为原料, 实现 了 $68.55 \%$ 的 $\mathrm{NaBH}_{4}$ 产率，简化了 $\mathrm{NaBO}_{2} \cdot x \mathrm{H}_{2} \mathrm{O}$ 脱水 和 $\mathrm{Mg}$ 氢化的过程, 为大幅降低 $\mathrm{NaBH}_{4}$ 的制备成本 提供了一种可能性。

\section{$1.2 \mathrm{NH}_{3} \cdot \mathrm{BH}_{3}$ 水解制氢}

相比 $\mathrm{NaBH}_{4}, \mathrm{NH}_{3} \cdot \mathrm{BH}_{3}$ 含氢量更高, 分子内部 的 $\mathrm{H}^{-} 、 \mathrm{H}^{+}$有较强的相互作用, 在加热到 $150{ }^{\circ} \mathrm{C}$ 时 能够生成 2 当量的 $\mathrm{H}_{2}{ }^{[40]}$, 在制氢领域极具吸引力, Demirci ${ }^{[41]}$ 综述了 $\mathrm{NH}_{3} \cdot \mathrm{BH}_{3}$ 的合成方法与放氢方式。 其水解制氢按式(4)所示的总反应进行。

$$
\mathrm{NH}_{3} \cdot \mathrm{BH}_{3}+2 \mathrm{H}_{2} \mathrm{O}=\mathrm{NH}_{4} \cdot \mathrm{BO}_{2}+3 \mathrm{H}_{2}
$$

水解过程只能夺取与 $\mathrm{B}$ 成键的 3 个 $\mathrm{H}$, 因此, 按 水解方式制氢, $\mathrm{NH}_{3} \cdot \mathrm{BH}_{3}$ 的理论制氢量少于 $\mathrm{NaBH}_{4}$ 。 它的合成又以 $\mathrm{NaBH}_{4}$ 为原料, 成本极高。此外, 其 水解副产物中含有 $\mathrm{NH}_{3} \cdot \mathrm{H}_{2} \mathrm{O}$, 所制备的 $\mathrm{H}_{2}$ 可能混有 $\mathrm{NH}_{3}$ 。与 $\mathrm{NaBH}_{4}$ 相比, $\mathrm{NH}_{3} \cdot \mathrm{BH}_{3}$ 的其他性能也无明 显优势。综上所述, 虽然 $\mathrm{NH}_{3} \cdot \mathrm{BH}_{3}$ 具有极高的含氢 量, 但是用于水解制氢，应用前景不如 $\mathrm{NaBH}_{4}$ 。

$\mathrm{NH}_{3} \cdot \mathrm{BH}_{3}$ 的催化水解机理近年来取得不错的进 展。曾杰课题组 ${ }^{[42]}$ 利用单原子分散的 $\mathrm{Rh} / \mathrm{VO}_{2}$ 催化 剂, 研究在氨硼烷水解过程中催化剂 $\mathrm{Rh}$ 的最高占 据态对反应活化能的影响。在 $341 \mathrm{~K}$ 时基底 $\mathrm{VO}_{2}$ 的 单斜-金红石的相转变会导致绝缘-导电性的转变, 进而影响 $\mathrm{Rh}$ 单原子的电子占据态。不同的电子密 度催化活性不同。如图 3 所示 ${ }^{[42]}$, 态密度计算结果 与测得的反应活化能转变温度高度契合, 验证了上 述机理。

陈勇课题组 ${ }^{[43}$ 通过共沉淀法和固相反应制备 的 Ni-Co-P/GO 三元催化剂, 是一种高活性的非贵 金属催化剂, 标准状态下 TOF(Time of frequency)值 高达 $513.9 \mathrm{~mol}_{\mathrm{H}_{2}} \cdot \mathrm{mol}^{-1}$ cat $\mathrm{min}^{-1}$ 。他们通过 X 射线吸 收光谱研究了三元催化剂中掺入 Co 以及氧化石 


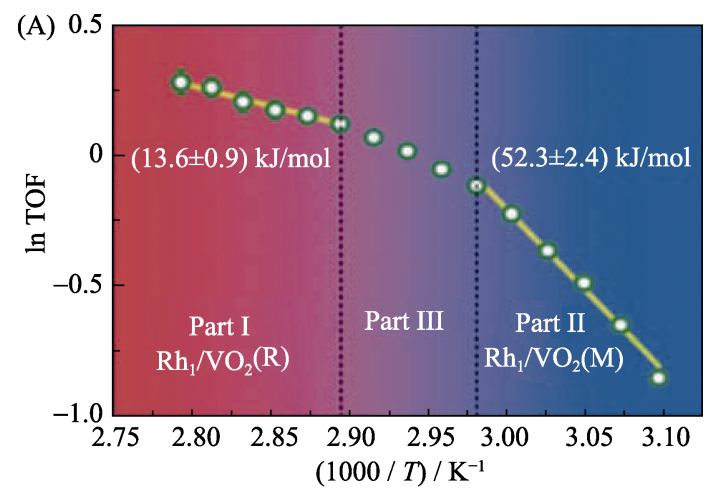

(B)

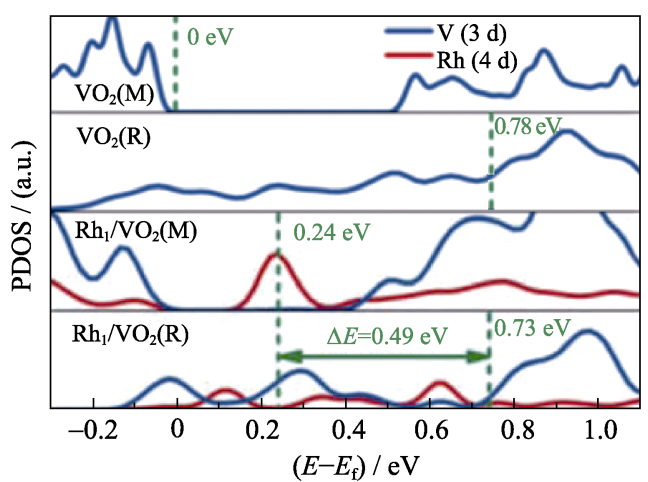

图 $3 \mathrm{Rh} / \mathrm{VO}_{2}$ 催化 $\mathrm{NH}_{3} \cdot \mathrm{BH}_{3}$ 水解的 Arrhenius 曲线(A), 不同 催化剂和基底的态密度计算结果(B) ${ }^{[42]}$

Fig. 3 Arrhenius plot for $\mathrm{NH}_{3} \cdot \mathrm{BH}_{3}$ hydrolysis catalyzed by $\mathrm{Rh} / \mathrm{VO}_{2}(\mathrm{~A})$, calculated PDOS of several catalysts/supports $(\mathrm{B})^{[42]}$

墨烯(GO)载体对 $\mathrm{Ni}$ 电子结构的影响。掺入 $\mathrm{Co}$ 取代 了部分 $\mathrm{Ni}_{2} \mathrm{P}$ 中的 $\mathrm{Ni}$ 原子, 使得 $\mathrm{Ni}$ 周围的电子流向 $\mathrm{P}, \mathrm{Ni}$ 元素的价态升高, 从而增强了对 $\mathrm{NH}_{3} \cdot \mathrm{BH}_{3}$ 的吸 附, 削弱了反应能垒。GO 载体能使催化剂更好地分 散, 还可以吸引催化剂上的电子, 改变催化剂的电 子结构, 提升其催化能力。

\section{2 金属水解制氢材料}

金属水解制氢材料最大的优势是原料来源广泛， 成本低廉, 规模制备工艺成熟。如表 1 所示, 有潜 力的金属水解制氢材料集中在元素周期表左上角。 碱金属保存条件苛刻，与水反应十分剧烈，除 $\mathrm{Li}$ 之 外, $\mathrm{Na} 、 \mathrm{~K}$ 的理论制氢量并无优势，一般不用于水解 制氢。研究较多的水解制氢材料为金属 $\mathrm{Mg}$ 和金属 $\mathrm{Al}$, 它们所共同面临的主要问题是表面钝化, 下面 分别进行介绍。

\section{1 金属 $\mathrm{Mg}$ 水解制氢}

金属 $\mathrm{Mg}$ 的水解往往需要在热水中进行, 如反 应式(5)所示。

$$
\mathrm{Mg}+2 \mathrm{H}_{2} \mathrm{O}=\mathrm{Mg}(\mathrm{OH})_{2}+\mathrm{H}_{2}
$$

消去 $\mathrm{Mg}$ 钝化层的方式包括: 加入 Brønsted 酸 $^{[44]}$ 、减小颗粒尺寸 ${ }^{[45]}$ 、合金化 ${ }^{[46]}$ 以及加入强酸弱
碱盐 ${ }^{[47-49]}$ 。加酸能很好地去除针化层, 但酸作为反 应物改变了水解反应路径, 使体系理论制氢量大大 减少。引入酸对设备耐腐蚀性的要求提升, 增加了 设备成本。减小 $\mathrm{Mg}$ 的颗粒尺寸能提升反应转化率, 但是过小的颗粒尺寸增加了原料加注的困难, 并且 发生爆炸的风险急剧上升。

加入强酸弱碱盐促进 $\mathrm{Mg}$ 水解是一种切实可行 且非常有效的方式。本课题组 ${ }^{[49]}$ 前期研究过不同阳 离子的氯盐对 $\mathrm{Mg}$ 水解的促进作用。如图 4 所示 ${ }^{[49]}$, 随着阳离子对 $\mathrm{OH}^{-}$亲和力的增大, 相应盐类对 $\mathrm{Mg}$ 水解的促进作用增强。通过弱碱性阳离子竞争结合 $\mathrm{OH}^{-}$, 加入盐能达到消除针化层的作用, 反应机理 可表示为式(6):

$$
n \mathrm{Mg}+2 \mathrm{M}^{n+}+2 n \mathrm{H}_{2} \mathrm{O}=n \mathrm{Mg}^{2+}+2 \mathrm{M}(\mathrm{OH})_{n}+n \mathrm{H}_{2}
$$

采用 $\mathrm{MgCl}_{2}$ 促进 $\mathrm{Mg}$ 水解, 溶液中的 $\mathrm{Mg}^{2+}$ 与 $\mathrm{Mg}$ 水解新产生的 $\mathrm{Mg}^{2+}$ 相互竞争 $\mathrm{OH}^{-}$, 水解过程中 溶液的 $\mathrm{Mg}^{2+}$ 总量几乎保存不变。少量 $\mathrm{MgCl}_{2}$ 能促进 大量的 $\mathrm{Mg}$ 持续水解, 是目前常用的一种手段。

\section{2 金属 $\mathrm{Al}$ 水解制氢}

金属 $\mathrm{Al}$ 是除 $\mathrm{NaBH}_{4}$ 外另一类被广泛研究的水解 制氢材料。 $\mathrm{Al}$ 是地壳中最丰富的金属元素, 电解铝工 艺成熟, 成本低廉, 具有广阔的应用前景。同时 $\mathrm{Al}$ 在 金属中具有较高的理论制氢量, 其水解按式(7)进行。

$$
2 \mathrm{Al}+6 \mathrm{H}_{2} \mathrm{O}=2 \mathrm{Al}(\mathrm{OH})_{3}+3 \mathrm{H}_{2}
$$

不同于 $\mathrm{Mg} 、 \mathrm{Al}$ 在反应之前其表面氧化形成的 针化层就能够阻止它与水反应，而在反应过程中的 钝化问题则与 $\mathrm{Mg}$ 类似。但 $\mathrm{Al}$ 作为两性金属, 反应 生成的氧化物及氢氧化物能够在酸性以及碱性溶液 中分别溶解, 形成 $\mathrm{Al}^{3+}$ 或者 $\mathrm{AlO}_{2}^{-}$。黄岳祥课题组 ${ }^{[50]}$ 总结了 $\mathrm{Al}$ 钝化层的几种主要消去方式, 包括碱促 进、合金化、盐促进和氧化物促进等。

修饰 $\mathrm{Al}$ 表面的氧化层是一种可取的方式, Deng

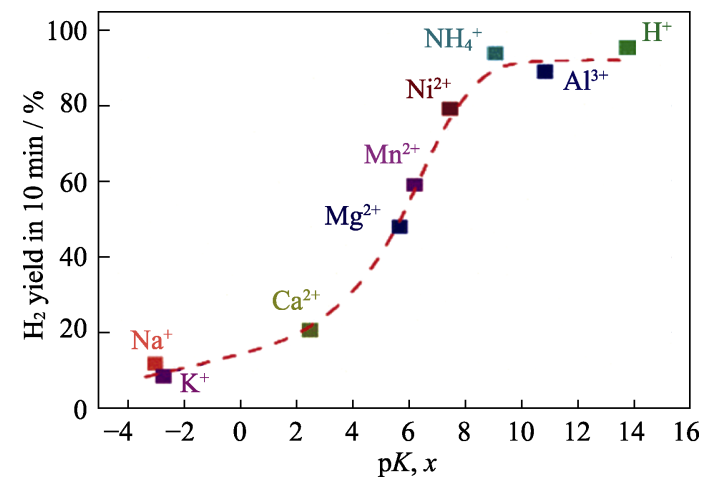

图 4 不同阳离子对于 $\mathrm{OH}^{-}$的亲和力与其促进 $\mathrm{Mg}$ 水解转化 率的关系 ${ }^{[49]}$

Fig. 4 Relationship between promotion of $\mathrm{Mg}$ hydrolysis and affinity to $\mathrm{OH}^{-}$for different cations ${ }^{[49]}$ 
等 ${ }^{[51]}$ 采用球磨法在 $\mathrm{Al}$ 表面构造了一层 $\gamma-\mathrm{Al}_{2} \mathrm{O}_{3}$ 以取 代 $\mathrm{Al}$ 表面本身形成的致密氧化膜。 $\gamma-\mathrm{Al}_{2} \mathrm{O}_{3}$ 能够和 水反应生成 $\mathrm{AlOOH}$, 转化完全时, $\mathrm{AlOOH}$ 层与内部 的 $\mathrm{Al}$ 接触，按式(8)反应。

$$
6 \mathrm{AlOOH}+2 \mathrm{Al}=4 \mathrm{Al}_{2} \mathrm{O}_{3}+3 \mathrm{H}_{2}
$$

产生的氢气气泡破坏 $\mathrm{Al}_{2} \mathrm{O}_{3}$ 和 $\mathrm{AlOOH}$ 的壳层后, $\mathrm{Al}$ 与水反应, 生成新的 $\mathrm{AlOOH}$, 并重复上述过程, 使 $\mathrm{Al}$ 几乎转化完全。

$\mathrm{Al}$ 与一些具有水解能力的金属合金化, 既能够 促进水解完全进行, 又能保证制氢量。最常采用的手 段是与 $\mathrm{Li}$ 合金化。严密课题组和王新华课题组 ${ }^{[52]}$ 将 $\mathrm{Al}$ 与 $\mathrm{LiH}$ 球磨混合, 在水解过程中生成 $\mathrm{LiAl}_{2}(\mathrm{OH})_{7}$ 和 $\mathrm{OH}^{-}$, 后者有助于 $\mathrm{Al}$ 水解持续进行。 $\mathrm{Al}$ 与 $\mathrm{LiH}$ 混合的水解材料能够实现 $1442.4 \mathrm{~mL}_{\mathrm{H}_{2}} \cdot \mathrm{g}^{-1}$ 的实际产 氢量。范美强等 ${ }^{[53-56]}$ 把镁铝合金与 $\mathrm{NaBH}_{4}$ 进行混合, 并加入少量过渡金属盐。合金与 $\mathrm{NaBH}_{4}$ 各自的水解 产物能相互促进水解。

黄岳祥课题组 ${ }^{[57]}$ 利用 $\mathrm{Al}$ 和 $\mathrm{Hg}$ 合金化, 构造了 一种特殊的 $\mathrm{Al}$ 水解制氢机制, 如图 5 所示 ${ }^{[57]}$, 他们 在 $\mathrm{Al}$ 棒料的上底面覆盖一层 $\mathrm{Hg}$ (或 $\mathrm{Zn}$ 永齐), 然后 用聚丙烯管将 $\mathrm{Al}$ 和 $\mathrm{Hg}$ 包裹住, 并在 $\mathrm{Hg}$ 上方加入 $\mathrm{H}_{2} \mathrm{O}$ 。 $\mathrm{Al}$ 逐渐溶解于 $\mathrm{Hg}$ 中, 与水进行反应, 实现了 稳定的产氢速率, $65{ }^{\circ} \mathrm{C}$ 下按该方式水解的产氢速率 稳定，可达 $43.5 \mathrm{~cm}^{3} \cdot \mathrm{h}^{-1} \cdot \mathrm{cm}^{-2}$ 。

\section{3 金属氢化物水解制氢材料}

金属氢化物水解制氢材料是金属吸氢后得到对 应的氢化物。若不计算水的质量与体积, 金属氢化 物的理论制氢量接近对应金属的两倍。在所有这类 材料中, 符合制氢量要求的是 $\mathrm{LiH} 、 \mathrm{MgH}_{2}$ 和 $\mathrm{AlH}_{3}$ 。 $\mathrm{LiH}$ 的保存和使用极其困难 ${ }^{[58]}$, 在 $5 \times 10^{-4} \mathrm{~mL} / \mathrm{mL}$ 的 $\mathrm{O}_{2}$ 中就会被氧化, 遇水容易发生燃烧。 $\mathrm{Al}$ 往往与其 它不同的金属形成混合型的金属氢化物，如 $\mathrm{LiAlH}_{4}$ 、 $\mathrm{Mg}\left(\mathrm{AlH}_{4}\right)_{2}$ 等, 单独的 $\mathrm{AlH}_{3}$ 的制备和保存 ${ }^{[59]}$ 也极其 困难。 $\mathrm{MgH}_{2}$ 的保存相对容易, 虽然现阶段制备成本 较高，但是其理论制氢量高，也被认为是有潜力的 水解制氢材料。本节主要介绍 $\mathrm{MgH}_{2}$ 水解制氢的研

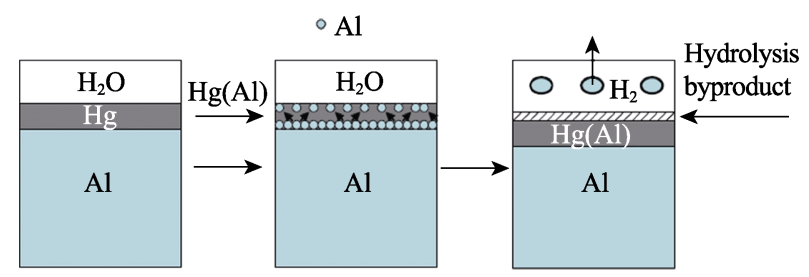

图 $5 \mathrm{Hg}$ 促进 $\mathrm{Al}$ 水解的构造和机理示意图 ${ }^{[57]}$

Fig. 5 Scheme of $\mathrm{Al}$ hydrolysis mechanism promoted by $\mathrm{Hg}^{[57]}$
究进展。

$\mathrm{MgH}_{2}$ 同样面临反应针化问题, 解决办法也基本 类似，主要采取 $\mathrm{MgCl}_{2}$ 促进的方式。包括本课题组在 内的多个课题组 ${ }^{[58,60-61]}$ 的研究表明 $\mathrm{MgCl}_{2}$ 对不同方 法制备得到的 $\mathrm{MgH}_{2}$ 水解都具有促进作用，其过程如 图 6 所示 ${ }^{[60]}$ 。Tegel 等 ${ }^{[62]}$ 系统研究了不同卤化物对于 $\mathrm{MgH}_{2}$ 水解的影响, 并提出了几种可能的促进机理, 与对金属本身的促进机理类似，包括： $\mathrm{pH}$ 降低; $\mathrm{Mg}(\mathrm{OH})_{2}$ 成核生长机制; 金属卤化物和 $\mathrm{Mg}(\mathrm{OH})_{2}$ 混 合沉淀。

与其他水解制氢材料混合也是一种较为有效的 提升 $\mathrm{MgH}_{2}$ 性能的方式。郡怀宇课题组和欧阳柳章 课题组 ${ }^{[46]}$ 采用熔融盐电解法制备了镁锂合金，通过 氢压球磨, 得到了以 $\mathrm{LiH}$ 和 $\mathrm{MgH}_{2}$ 为主的混合水解 材料。该材料的实际制氢量达到 $1773 \mathrm{~mL}_{\mathrm{H}_{2}} \cdot \mathrm{g}^{-1}$, 比 未氢化的镁锂合金的制氢量提升了近 1 倍。

$\mathrm{MgH}_{2}$ 的制备也是限制其发展的主要因素之一, 主要原因是 $\mathrm{Mg}$ 吸氢缓慢。上海交通大学的邹建新 和邬剑波课题组 ${ }^{[63]}$ 运用等离子体电弧法、固相法制 备了表面 Pt 分散的 $\mathrm{Mg}$ 纳米晶，其形貌如图 7 所示。 该结构的纳米颗粒通过氢溢流效应构筑了“氢泵”, 将 $\mathrm{Mg}$ 在低温条件下的吸放氢动力学相比普通 $\mathrm{Mg}$ 纳米颗粒提升了约 $30 \%$ 。虽然贵金属的引入会进一 步提升 $\mathrm{MgH}_{2}$ 的成本, 但是构筑氢百的机制值得借 鉴。进一步开发 Pt 的廉价替代品构筑氢百，预计能 降低成本并显著提升 $\mathrm{MgH}_{2}$ 材料水解制氢性能。

\section{4 总结与展望}

综上所述，水解制氢拥有不可替代的储氢密度

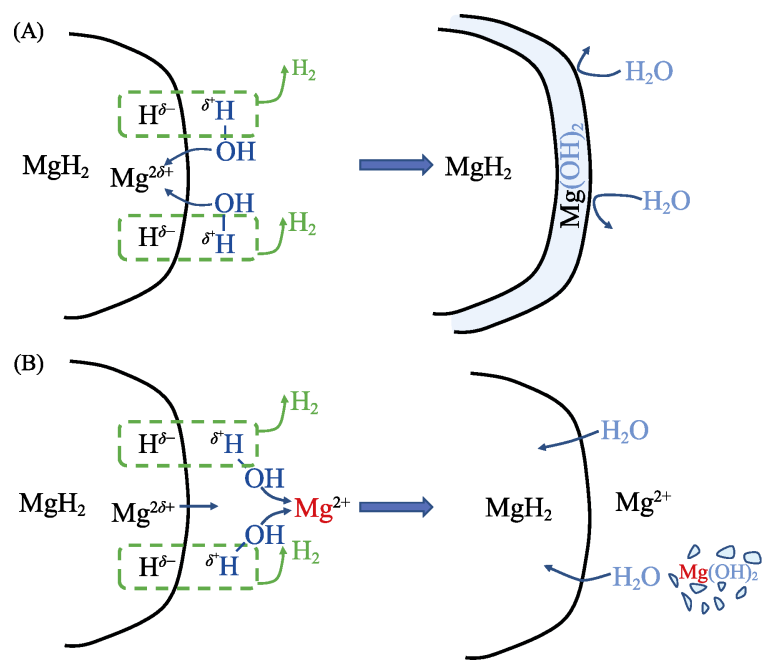

图 $6 \mathrm{MgH}_{2}$ 在去离子水 $(\mathrm{A})$ 和 $\mathrm{MgCl}_{2}$ (B) 中的反应机理 ${ }^{[60]}$ Fig. 6 Schemes of $\mathrm{MgH}_{2}$ hydrolysis mechanisms in $\mathrm{H}_{2} \mathrm{O}$ (A) and $\mathrm{MgCl}_{2}$ (aq.) (B) ${ }^{[60]}$ 


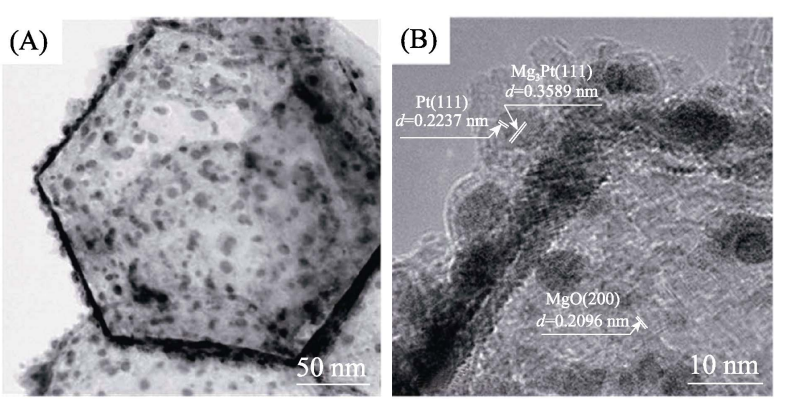

图 7 吸氢过后 $\mathrm{Mg} @ \mathrm{Pt}$ 的透射电镜照片 $(\mathrm{A})$ 与高分辨透射电 镜照片 $(\mathrm{B})^{[63]}$

Fig. 7 TEM (A) and HRTEM (B) images of hydrogenated $\mathrm{Mg} @$ Pt composite ${ }^{[63]}$

\section{表 2 主要制氢材料性能对比}

Table 2 Performances of main materials for hydrogen production via hydrolysis

\begin{tabular}{|c|c|c|c|}
\hline Performance & Metal & Metal hydride & Borohydride \\
\hline $\begin{array}{c}\text { Hydrogen capacity } \\
\text { of hydrolysis }\end{array}$ & Low & High & $\begin{array}{l}\text { High (affected } \\
\text { by dissolution) }\end{array}$ \\
\hline Cost & Low & High & High \\
\hline $\begin{array}{l}\text { Complexities } \\
\text { of system }\end{array}$ & Complex & Complex & $\begin{array}{l}\text { Relatively } \\
\text { simple }\end{array}$ \\
\hline $\begin{array}{l}\text { Degree of study } \\
\text { on hydrolysis }\end{array}$ & Modest & Rare & Extensive \\
\hline Storage condition & $\begin{array}{l}\mathrm{H}_{2} \mathrm{O} \& \\
\mathrm{O}_{2} \text { free }\end{array}$ & $\begin{array}{l}\mathrm{H}_{2} \mathrm{O} \& \\
\mathrm{O}_{2} \text { free }\end{array}$ & $\mathrm{H}_{2} \mathrm{O}$ free \\
\hline
\end{tabular}

\section{表 3 常见水解制氢材料的价格及制氢成本估算}

Table 3 Prices of materials for hydrogen generation via hydrolysis and their corresponding costs of $\mathbf{H}_{2}$

\begin{tabular}{ccccccc}
\hline Prices & $\mathrm{Mg}$ & $\mathrm{Al}$ & $\mathrm{MgH}_{2}$ & $\mathrm{LiH}$ & $\mathrm{NaBH}_{4}$ & $\mathrm{NH}_{3} \cdot \mathrm{BH}_{3}$ \\
\hline $\begin{array}{c}\text { Price of material } \\
/\left(\text { yuan } \cdot \mathrm{kg}^{-1}\right)\end{array}$ & 120 & 80 & 800 & 4300 & 700 & 60000 \\
$\begin{array}{c}\text { Price of } \mathrm{H}_{2} \\
/\left(\text { yuan } \cdot \mathrm{kg}^{-1}\right)\end{array}$ & 1450 & 710 & 5200 & 16960 & 3280 & 307700 \\
\hline
\end{tabular}

Prices of materials are based on particle materials at reagent grade; Prices of $\mathrm{H}_{2}$ are estimated by the prices of materials, which are lower than the real cost

高、制氢条件温和、储存运输便捷等优势，具有广 阔的应用前景。目前主要对中小型燃料电池供氢, 应 用场景包括 AIP(Air Independent Propulsion)动力潜 艇 ${ }^{[9]}$ 、无人潜航器、固定翼的无人机 ${ }^{[7-8]}$ 以及野外单 兵作战装备 ${ }^{[64]}$ 等。水解制氢在上述领域具有其他制 氢方式无法替代的优势。本文介绍了三类主要的水 解制氢材料, 包括嗍氢化物 $\left(\mathrm{NaBH}_{4}, \mathrm{NH}_{3} \cdot \mathrm{BH}_{3}\right)$ 、金属 $(\mathrm{Mg}, \mathrm{Al})$ 和金属氢化物 $\left(\mathrm{MgH}_{2}\right)$, 它们各自的特点优势 见表 2。

由于高成本的原料(表 3)和复杂的体系, 水解制 氢短期内难以应用到车载动力电池领域, 后续如果 在制备方法上有所突破, 成本降至与锂离子电池甚 至传统化石燃料相当, 则有望应用于车载和分布式
燃料电池供电系统中。作者认为要实现水解制氢的 实用化和产业化, 未来的研究还需要在以下几个方 面进一步探索:

1)开发新的水解制氢体系，合成新的水解材料 及催化剂，设计新型的制氢方式和促进机制，实现 综合性能良好的高含氢量水解制氢。

2)与其他能源形式进行有效地复合, 实现更加 高效的储能模式。

3)探索新的合成方法，进一步降低高含氢量水 解制氢材料的成本。

4)通过材料设计实现水解制氢过程的控制，提 升反应的稳定性和可控性，降低工程复杂度。

5)进行更加深入的理论研究, 包括钝化层消去 过程和不同催化剂异相催化的机理等, 加深对反应 过程的认识, 以实现更精准的调控。

6)根据水解反应的特点, 寻找水解制氢的非储 能新应用。

\section{参考文献:}

[1] EBERLE U, FELDERHOFF M, SCHUTH F. Chemical and physical solutions for hydrogen storage. Angew. Chemie. Int. Ed., 2009, 48(36): 6608-6630.

[2] HE T, PACHFUlE P, WU H, et al. Hydrogen carriers. Nat. Rev. Mater., 2016, 1: 16059.

[3] SCHLAPBACH L, ZÜTTEL A. Hydrogen-storage materials for mobile applications. Nature, 2001, 414: 353-358.

[4] GÜR T M. Review of electrical energy storage technologies, materials and systems: challenges and prospects for large-scale grid storage. Energy \& Environ. Sci., 2018, 11(10): 2696-2767.

[5] STAMENKOVIC V R, STRMCNIK D, LOPES P P, et al. Energy and fuels from electrochemical interfaces. Nat. Mater., 2016, 16: 57-69.

[6] DEAN J A. Lange's Handbook of Chemistry, $13^{\text {th }}$ Edition. Beijing: Sci. Press, 1991, 9: 1-172.

[7] KIM H, OH T H, KWON S J. Simple catalyst bed sizing of a $\mathrm{NaBH}_{4}$ hydrogen generator with fast startup for small unmanned aerial vehicles. Int. J. Hydrogen Energy, 2016, 41(2): 1018-1026.

[8] KIM T. $\mathrm{NaBH}_{4}$ (sodium borohydride) hydrogen generator with a volume-exchange fuel tank for small unmanned aerial vehicles powered by a PEM (proton exchange membrane) fuel cell. Energy, 2014, 69: 721-727.

[9] LI H W, LI D P, ZHANG X D. Research and development of foreign submarine sodium borohydride hydrolysis hydrogen generation. Ship Sci. Technol., 2012, 34(7): 135-143.

[10] OH T K. Conceptual design of small unmanned aerial vehicle with proton exchange membrane fuel cell system for long endurance mission. Energy Convers. Manag., 2018, 176: 349-356.

[11] CHEN K, OUYANG L Z, ZHONG $\mathrm{H}$, et al. Converting $\mathrm{H}^{+}$from coordinated water into $\mathrm{H}^{-}$enables super facile synthesis of $\mathrm{LiBH}_{4}$. Green Chem., 2019, 21(16): 4380-4387.

[12] SEN B, ŞAVK A, KUYULDAR E, et al. Hydrogen liberation from the hydrolytic dehydrogenation of hydrazine borane in acidic media. Int. J. Hydrogen Energy, 2018, 43(38): 17978-17983.

[13] KAUFMAN C M, SEN B. Hydrogen generation by hydrolysis of sodium tetrahydroborate: effects of acids and transition metals and 
their salts. Dalton Trans., 1984, (2): 307-313.

[14] LIU B H, LI Z P. A review: hydrogen generation from borohydride hydrolysis reaction. J. Power Sources, 2009, 187(2): 527-534.

[15] BOZKURT G, ÖZER A, YURTCAN A B. Development of effective catalysts for hydrogen generation from sodium borohydride: $\mathrm{Ru}, \mathrm{Pt}$, Pd nanoparticles supported on $\mathrm{Co}_{3} \mathrm{O}_{4}$. Energy, 2019, 180: 702-713.

[16] GENÇ A E, AKÇA A, KUTLU B. The catalytic effect of the $\mathrm{Au}(111)$ and $\mathrm{Pt}(111)$ surfaces to the sodium borohydride hydrolysis reaction mechanism: a DFT study. Int. J. Hydrogen Energy, 2018, 43(31): 14347-14359.

[17] SEMIZ L, ABDULlAYEVA N, SANKIR M. Nanoporous Pt and $\mathrm{Ru}$ catalysts by chemical dealloying of Pt-Al and $\mathrm{Ru}-\mathrm{Al}$ alloys for ultrafast hydrogen generation. J. Alloys Compd., 2018, 744(5): $110-115$.

[18] TUAN D D, LIN K Y A. Ruthenium supported on ZIF-67 as an enhanced catalyst for hydrogen generation from hydrolysis of sodium borohydride. Chem. Eng. J., 2018, 351: 48-55.

[19] LI Y H, ZHANG X, ZHANG Q, et al. Activity and kinetics of ruthenium supported catalysts for sodium borohydride hydrolysis to hydrogen. RSC Adv., 2016, 6(35): 29371-29377.

[20] HAO S, YANG L B, CUI L, et al. Self-supported spinel $\mathrm{FeCo}_{2} \mathrm{O}_{4}$ nanowire array: an efficient non-noble-metal catalyst for the hydrolysis of $\mathrm{NaBH}_{4}$ toward on-demand hydrogen generation. Nanotechnology, 2016, 27(46): 0957-4484.

[21] CAI H, LIU L, CHEN Q, et al. Ni-polymer nanogel hybrid particles: a new strategy for hydrogen production from the hydrolysis of dimethylamine-borane and sodium borohydride. Energy, 2016, 99: 129-135.

[22] LUO C, FU F Y, YANG X J, et al. Highly efficient and selective Co@ZIF-8 nanocatalyst for hydrogen release from sodium borohydride hydrolysis. ChemCatChem, 2019, 11(6): 1643-1649.

[23] MAHMOOD J, JUNG S M, KIM S J, et al. Cobalt oxide encapsulated in $\mathrm{C}_{2} \mathrm{~N}-\mathrm{h} 2 \mathrm{D}$ network polymer as a catalyst for hydrogen evolution. Chem. Mater., 2015, 27(13): 4860-4864.

[24] LIN K Y A, CHANG H A, CHEN B J. Multi-functional MOF-derived magnetic carbon sponge. J. Mater. Chem. A, 2016, 4(35): 13611-13625.

[25] GUO S Q, WU Q Q, SUN J, et al. Highly stable and controllable $\mathrm{CoB} / \mathrm{Ni}$-foam catalysts for hydrogen generation from alkaline $\mathrm{NaBH}_{4}$ solution. Int. J. Hydrogen Energy, 2017, 42(33): 21063-21072.

[26] CUI L, SUN X P, XU Y H, et al. Cobalt carbonate hydroxide nanowire array on Ti mesh: an efficient and robust 3D catalyst for on-demand hydrogen generation from alkaline $\mathrm{NaBH}_{4}$ solution. Chem. Eur. J., 2016, 22(42): 14831-14835.

[27] PATEL N, MiOTEllo A. Progress in Co-B related catalyst for hydrogen production by hydrolysis of boron-hydrides: a review and the perspectives to substitute noble metals. Int. J. Hydrogen Energy, 2015, 40(3): 1429-1464.

[28] GUO M S, CHENG Y, YU Y N, et al. Ni-Co nanoparticles immobilized on a 3D Ni foam template as a highly efficient catalyst for borohydride electrooxidation in alkaline medium. Appl. Surf. Sci., 2017, 416: 439-445.

[29] MARCHIONNI A, BEVILACQUA M, FILIPPI J, et al. High volume hydrogen production from the hydrolysis of sodium borohydride using a cobalt catalyst supported on a honeycomb matrix. J. Power Sources, 2015, 299(20): 391-397.

[30] ZHUANG D W, DAI H B, ZHONG Y J, et al. A new reactivation method towards deactivation of honeycomb ceramic monolith supported cobalt-molybdenum-boron catalyst in hydrolysis of sodium borohydride. Int. J. Hydrogen Energy, 2015, 40(30): 9373-9381.
[31] SAHINER N. Soft and flexible hydrogel templates of different sizes and various functionalities for metal nanoparticle preparation and their use in catalysis. Prog. Polym. Sci., 2013, 38(9): 1329-1356.

[32] Ai L H, GAO X Y, JIANG J. In situ synthesis of cobalt stabilized on macroscopic biopolymer hydrogel as economical and recyclable catalyst for hydrogen generation from sodium borohydride hydrolysis. J. Power Sources, 2014, 257(1): 213-220.

[33] PRASAD D, PATIL K N, CHAITRA C R, et al. Sulfonic acid functionalized PVA/PVDF composite hollow microcapsules: highly phenomenal \& recyclable catalysts for sustainable hydrogen production. Appl. Surf. Sci., 2019, 488: 714-727.

[34] LI Q M, CHEN Y B, LEE D J, et al. Preparation of Y-zeolite/ $\mathrm{CoCl}_{2}$ doped PVDF composite nanofiber and its application in hydrogen production. Energy, 2012, 38(1): 144-150.

[35] ZHAO L W, LI Q, SU Y, et al. A novel enteromorpha based hydrogel for copper and nickel nanoparticle preparation and their use in hydrogen production as catalysts. Int. J. Hydrogen Energy, 2017, 42(10): 6746-6756.

[36] SCHLESINGER H I, BROWN H C, FINHOLT A E. The preparation of sodium borohydride by the high temperature reaction of sodium hydride with borate esters. J. Am. Chem. Soc., 1953, 75: 205-209.

[37] KOJIMA Y, HAGA T. Recycling process of sodium metaborate to sodium borohydride. Int. J. Hydrogen Energy, 2003, 28: 989-993.

[38] LANG C G, JIA Y, LIU J W, et al. $\mathrm{NaBH}_{4}$ regeneration from $\mathrm{NaBO}_{2}$ by high-energy ball milling and its plausible mechanism. Int. J. Hydrogen Energy, 2017, 42(18): 13127-13135.

[39] OUYANG L Z, CHEN W, LIU J W, et al. Enhancing the regeneration process of consumed $\mathrm{NaBH}_{4}$ for hydrogen storage. Adv. Energy Mater, 2017, 7(19): 1700299.

[40] SANYAL U, DEMIRCI U B, JAGIRDAR B R, et al. Hydrolysis of ammonia borane as a hydrogen source: fundamental issues and potential solutions towards implementation. ChemSusChem, 2011, 4(12): 1731-1739.

[41] DEMIRCI U B. Ammonia borane, a material with exceptional properties for chemical hydrogen storage. Int. J. Hydrogen Energy, 2017, 42(15): 9978-10013.

[42] WANG L B, LI H L, ZHANG W B, et al. Supported rhodium catalysts for ammonia-borane hydrolysis: dependence of the catalytic activity on the highest occupied state of the single rhodium atoms. Angew. Chemie. Int. Ed., 2017, 56(17): 4712-4718.

[43] HOU C C, LI Q, WANG C J, et al. Ternary Ni-Co-P to boost the hydrolytic dehydrogenation of ammonia-borane. Energy \& Environ. Sci., 2017, 10(8): 1770-1776.

[44] PIGHIN S A, URRETAVIZCAYA G, BOBET J L. Nanostructured $\mathrm{Mg}$ for hydrogen production by hydrolysis obtained by $\mathrm{MgH}_{2}$ milling and dehydriding. J. Alloys Compd., 2020, 827: 154000.

[45] TAN Z H, OUYANG L Z, LIU J W, et al. Hydrogen generation by hydrolysis of $\mathrm{Mg}-\mathrm{Mg}_{2} \mathrm{Si}$ composite and enhanced kinetics performance from introducing of $\mathrm{MgCl}_{2}$ and Si. Int. J. Hydrogen Energy, 2018, 43(5): 2903-2912.

[46] JIANG J, OUYANG L Z, WANG H, et al. Controllable hydrolysis performance of $\mathrm{MgLi}$ alloys and their hydrides. ChemPhysChem, 2019, 20(10): 1316-1324.

[47] GAN D Y, LIU Y N, ZHANG J G, et al. Kinetic performance of hydrogen generation enhanced by $\mathrm{AlCl}_{3}$ via hydrolysis of $\mathrm{MgH}_{2}$ prepared by hydriding combustion synthesis. Int. J. Hydrogen Energy, 2018, 43(22): 10232-10239.

[48] ZHAO Z L, ZHU Y F, LI L Q. Efficient catalysis by $\mathrm{MgCl}_{2}$ in hydrogen generation via hydrolysis of Mg-based hydride prepared by hydriding combustion synthesis. Chem. Comm., 2012, 48(44): 5509-5511. 
[49] ZHENG J, YANG D C, LI W, et al. Promoting $\mathrm{H}_{2}$ generation from the reaction of $\mathrm{Mg}$ nanoparticles and water using cations. Chem. Comm., 2013, 49(82): 9437-9439.

[50] HUANG X N, GAO T, PAN X L, et al. A review: feasibility of hydrogen generation from the reaction between aluminum and water for fuel cell applications. J. Power Sources, 2013, 229: 133-140.

[51] DENG Z Y, FERREIRA J M F, TANAKA Y, et al. Physicochemical mechanism for the continuous reaction of $\gamma-\mathrm{Al}_{2} \mathrm{O}_{3}$-modified aluminum powder with water. $J$. Am. Chem. Soc., 2007, 90(5): $1521-1526$.

[52] LIU Y A, WANG X H, LIU H Z, et al. Improved hydrogen generation from the hydrolysis of aluminum ball milled with hydride. Energy, 2014, 72: 421-426.

[53] FAN M Q, WANG Y, TIAN G L, et al. Hydrolysis of AlLi/ $/ \mathrm{NaBH}_{4}$ system promoted by Co powder with different particle size and amount as synergistic hydrogen generation for portable fuel cell. Int. J. Hydrogen Energy, 2013, 38(25): 10857-10863.

[54] FAN M Q, LIU S, SUN W Q, et al. Controllable hydrogen generation and hydrolysis mechanism of $\mathrm{AlLi} / \mathrm{NaBH}_{4}$ system activated by $\mathrm{CoCl}_{2}$ solution. Renew. Energ., 2012, 46: 203-209.

[55] FAN M Q, LIU S, SUN W Q, et al. Hydrogen generation from $\mathrm{Al} / \mathrm{NaBH}_{4}$ hydrolysis promoted by $\mathrm{Li}-\mathrm{NiCl}_{2}$ additives. Int. J. Hydrogen Energy, 2011, 36(24): 15673-15680.

[56] FAN M Q, WANG Y, TANG R, et al. Hydrogen generation from $\mathrm{Al} / \mathrm{NaBH}_{4}$ hydrolysis promoted by Co nanoparticles and $\mathrm{NaAlO}_{2}$ solution. Renew. Energ., 2013, 60: 637-642.

[57] HUANG X N, LV C J, HUANG Y X, et al. Effects of amalgam on hydrogen generation by hydrolysis of aluminum with water. Int. $J$. Hydrogen Energy, 2011, 36(23): 15119-15124.

[58] REN R M, ORTIZ A L, MARKMAITREE T, et al. Stability of lithium hydride in argon and air. J. Phys. Chem. B, 2006, 110(21): 10567-10575.

[59] DUAN C W, HU L X, MA J L. Ionic liquids as an efficient medium for the mechanochemical synthesis of $\alpha-\mathrm{AlH}_{3}$ nano-composites. $J$. Mater. Chem. A, 2018, 6(15): 6309-6318.

[60] CHEN J, FU H, XIONG Y F, et al. $\mathrm{MgCl}_{2}$ promoted hydrolysis of $\mathrm{MgH}_{2}$ nanoparticles for highly efficient $\mathrm{H}_{2}$ generation. Nano Energy, 2014, 10: 337-343.

[61] HUANG M H, OUYANG L Z, WANG H, et al. Hydrogen generation by hydrolysis of $\mathrm{MgH}_{2}$ and enhanced kinetics performance of ammonium chloride introducing. Int. J. Hydrogen Energy, 2015, 40(18): 6145-6150.

[62] TEGEL M, SCHÖNE S, KIEBACK B, et al. An efficient hydrolysis of $\mathrm{MgH}_{2}$-based materials. Int. J. Hydrogen Energy, 2017, 42(4): 2167-2176.

[63] LU C, MA Y L, LI F, et al. Visualization of fast "hydrogen pump" in core-shell nanostructured $\mathrm{Mg} @ \mathrm{Pt}$ through hydrogen-stabilized Mg 3 Pt. J. Mater. Chem. A, 2019, 7(24): 14629-14637.

[64] SIFER N, GARDNER K. An analysis of hydrogen production from ammonia hydride hydrogen generators for use in military fuel cell environments. J. Power Sources, 2004, 132: 135-138. 